\title{
Network Security Analysis in Vanet Against Black Hole and Jellyfish Attack with Intrusion Detection System Algorithm
}

\author{
Elsa Mustikawati ${ }^{1}$, Doan Perdana ${ }^{2}$, and Ridha Muldina Negara ${ }^{3}$ \\ ${ }^{1,2,3}$ Faculty of Electrical Engineering, Telkom University, Bandung 40257, Indonesia \\ Email: ${ }^{1}$ elsamustikawati@gmail.com, ${ }^{2}$ elsamustikawati@gmail.com , \\ 3 ridhanegara@telkomuniversity.ac.id
}

\begin{abstract}
VANET is the key to the Intelligent Transportation Systems (ITS), where vehicles can communicate with others to exchange information in real time. VANET is an ad-hoc that has no fixed infrastructure and rapidly changing network topology. As the result, the network is insecure and vulnerable to various attacks both from within and outside the network. This research analyzes AODV routing protocol comparing the conditions without the attacks and with the attacks with the of black hole and jellyfish using the algorithm of Intrusion Detection System (IDS) with the number of nodes changing from 10 to 100 nodes at the change speeds of $70,80, \ldots$, 110 , and $120 \mathrm{~km} / \mathrm{h}$. This research is simulated using Network Simulator 2 to model the network and ONE Simulator to model node mobility. The analyzed QoS parameters are Packet Delivery Ratio (PDR), throughput, and end-to-end delay. The results of the simulation show that changing the number of nodes and node velocity affects the performance in the network. On the number of nodes scenario with attacks, the average value of PDR decreases by $48.03 \%$, throughput decreases by $50.23 \%$, and delay, for black hole, decreases by $80.18 \%$ but increases by $47.87 \%$ for jellyfish. Whereas in the node velocity scenario, the average values of PDR, throughput, and delay decrease by $58.52 \%, 60.34 \%, 13.81 \%$ for black hole attack, respectively. However, the delay increases by $\mathbf{1 2 3 . 9 1 \%}$ for jellyfish attack.
\end{abstract}

Index Terms-Black Hole Attack, IDS, Jellyfish Attack, and VANET

\section{INTRODUCTION}

V EHICULAR Ad Hoc Network (VANET) is an adhoc network that allows vehicles to communicate with others without any fixed infrastructure. VANETs is a promising approach to Intelligent Transportation System (ITS) [1]. A network system that connects to the Internet requires better protection in usage because it has a high threat level [2]. Ad-hoc network has a decentralized architecture, and algorithm in ad-hoc

Received: Aug. 9, 2017; received in revised form: Aug. 25, 2017; accepted: Oct. 30, 2017; available online: Nov. 28, 2017. network relies on the participation of cooperative node on the network VANET. Thus, any decision making is decentralized. It can be used by attackers to perform the attack. The attack aims to destabilize a running cooperative algorithm [3]. It makes VANET network vulnerable to attacks that can cause problems even on small networks. In addition, it can pose a threat to network security that can worsen the function or network services. Among all the existing challenges, network security on VANET is less noted for this. Data packets in VANET contain critical information and need to be ensured that those packages are not accessed or modified by attackers. Network security issue is not similar to the communication network in general. The size of the network, mobility, geographic relevance, and other things make this implementation difficult and different from other network security [4].

Reference [5] studies that black hole attack reduces packet delivery fraction to $10 \%-40 \%$, but when there are the addition of Intrusion Detection System (IDS) algorithm, it is up to $90 \%-98 \%$. The IDS algorithm has the advantage of not requiring the addition of overhead and slight modification of AODV. Reference [6] simulates jellyfish attack using AODV routing protocol on a network of MANET. The results of these simulations indicate that the jellyfish affects network performance with improving end-to-end delay and jitter. Based on previous related research, it can be concluded that the offensive black hole and jelly fish can be solved by modifying the used routing protocol, namely AODV. Because some attacks carry out the modifications to the RREP message that interferes with the process of routing. It counterfeits RREP message in which malicious nodes have the latest and fastest routing.

In this research, to prevent the attacks, IDSAODV is used as prevention algorithm for both attacks in two scenarios. The vehicular environment is modeled in 
Cite this article as: E. Mustikawati, D. Perdana, and R. M. Negara, "Network Security Analysis in Vanet Against Black Hole and Jelly Fish Attack with Intrusion Detection System Algorithm”, CommIT (Communication \& Information Technology) Journal 11(2), 77-83, 2017.

highway road, Jakarta-Cikampek toll road.

\section{LITERATURE REVIEW}

\section{A. Vehicular Ad-Hoc Network}

VANET is wireless ad-hoc that allows communication between vehicles or vehicles with devices on the roadside. It enhances the security of transportation. The movement of the vehicles or node on VANET is very dynamic because the vehicle is moving at a high speed and the position changes constantly. These characteristics make network topology change rapidly, so the link between node connect and disconnect very often [7].

The communication mode on VANET can be classified into two categories. Vehicle-to-vehicle (V2V) communications mode is between the nodes on the vehicle or On-Board Unit (OBU). Vehicle-toinfrastructure (V2I) road is between nodes or vehicles with hardware on the roadside or Road Side Unit (RSU) [7].

\section{B. Ad-Hoc on Demand Distance Vector}

Ad-hoc On Demand Distance Vector (AODV) routing protocol is the reactive and on-demand routing protocol. The routing table will only update when the source and destination nodes need to transmit data packets. AODV routing protocol operates in two stages, namely, route discovery and route maintenance [5]. When the source node wants to communicate with the destination node, but it does not have the route, then the source node will initialize the route discovery process. AODV route discovery process uses control messages to determine the route to the destination node. There are three control messages of route discovery as follows.

1) Route Request: Route Request (RREQ) will be sent when the source node wants to send a packet to the node that is not a node's neighbors which have not establish the route. Source node will broadcast the RREQ to all nodes, which is adjacent to the source node. Table I presents fields in RREP message. In the RREQ message, there is a hop count field that states the number of hops that must transmit by the RREQ. In addition, there is broadcast ID and a sequence number that serves to avoid the sending of the same message to a node.

2) Route Reply: When the nodes receive Route Reply (RREP) packets, the nodes will look up to its routing table whether it has a route to the destination or not. If the node has a route to the destination or the destination itself, it will send the RREP packets. When the RREP packet arrives on the source node, the route will be established through the intermediate nodes and

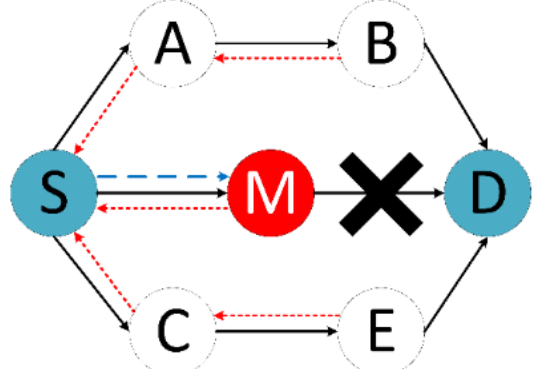

Fig. 1. Black hole attack mechanism.

path by assuming that it is the fastest route [8]. Table II is the fields inside RREP message.

\section{Black Hole Attack}

Black hole node sends false routing information by claiming that the node has the fastest route. By manipulating the RREP field using the highest sequence number, and setting hop count to 1 , it causes the intermediate nodes to send data packets to malicious nodes. After the route is established to black hole node, it will drop all the packets without forwarding the packet to the other nodes [9] (see Fig. 1).

\section{Jellyfish Attack}

Jellyfish attack can be classified into three subcategories. Jellyfish delay variance attack is applied in this research. Jellyfish node manipulates the other nodes to establish the route to itself by using false RREP. In this research, it is applied by changing the highest sequence number field and hop count is set to 1 . After the route is established, jellyfish node adds a delay on each packet before forwarding the packet, without changing the sequence of the packet. Delay can vary from 0 to $10 \mathrm{~s}$ randomly [6]. In this research, delay varies from 0 to $2 \mathrm{~s}$ randomly before forwarding the packet.

\section{E. Intrusion Detection System}

IDS is a system for detecting intrusion or interference on the network by collecting, analyzing and auditing system of the data on the network. IDS monitor activities continuously. For example traffic package is passed.

IDSAODV assumes that the first RREP message to come is RREP message from malicious nodes. In the route of discovery process, the first RREP message arrived is ignored and the second RREP is chosen to establish the routing path. This mechanism 
Cite this article as: E. Mustikawati, D. Perdana, and R. M. Negara, "Network Security Analysis in Vanet Against Black Hole and Jelly Fish Attack with Intrusion Detection System Algorithm”, CommIT

(Communication \& Information Technology) Journal 11(2), 77-83, 2017.

TABLE I

RREQ MESSAGE FIELD [8].

\begin{tabular}{llllll}
\hline Source_address & Source_sequence & Broadcast_id & Destination_address & Destination_sequence & Hop_count
\end{tabular}

TABLE II

RREQ MESSAGE FIELD [8].

\begin{tabular}{lllll}
\hline Source_address & Destination_address & Destination_sequence & Hop_count & Lifetime \\
\hline
\end{tabular}

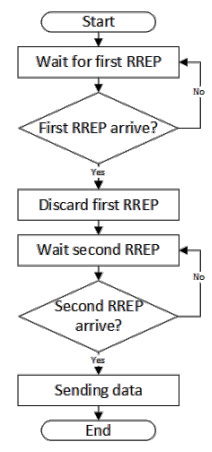

Fig. 2. Flowchart of RREP caching mechanism in IDSAODV.

is called RREP caching mechanism. In Network Simulator 2, four functions are added into idsaodv. Cc, which is rrep_insert to add the RREP message, rrep_lookup to check whether there is another RREP message, rrep_remove to remove all the records from the other until the RREP message from a particular node and rrep_purge to remove the RREP periodically if it has expired. IDSAODV will choose the second RREP arrived as the routing path, whether it comes from malicious node or not [10].

\section{RESEARCH METHOD}

To prevent black hole and jellyfish attack, IDSAODV is applied in preventing or reducing the effects of network performance. Inside IDSAODV, there are RREP caching mechanism to reduce the effects of the attack by ignoring the first RREP packet. Figure 2 shows RREP caching mechanism in IDSAODV. First, the node will wait for the first arrived RREP packet. If the first RREP packet has arrived, IDSAODV will ignore it and wait for the next RREP packet. The node will establish routing patch through the node that sends the next RREP packet.

This RREP caching mechanism assumes that the first RREP packet comes from the black hole or jellyfish node which contains false RREP. In the black hole and jellyfish attack, the malicious node will send false RREP that contains the highest sequence number and hop count is set to one. Thosel manipulate the
TABLE III

SimUlation PARAMETERS

\begin{tabular}{ll}
\hline Parameter & Detail \\
\hline Simulation Dimension & $8000 \times 1000$ \\
Simulasi Duration & 400 seconds \\
Node Density & 10 to 100 nodes \\
Node Velocity & $70,80,90,100,110,120 \mathrm{~km} / \mathrm{h}$ \\
Routing Protocol & AODV, idsAODV \\
Malicious Node & Single Blackhole or Single Jellyfish \\
Mobility Model & Map Based Movement \\
Traffic Direction & Two ways \\
MAC & MAC 802_11 Ext (IEEE 802.11p) \\
PHY & Wireless 802_11 Ext (IEEE 802.11p) \\
Propagation Wave Model & Two Ray Ground \\
Antenna Type & Omnidirectional \\
Transport Layer & UDP \\
Traffic Model & CBR \\
\hline
\end{tabular}

other nodes to establish routing patch to itself. With IDSAODV, the first RREP packet is ignored and it reduces the chances to establish the routing path to the malicious node.

Mobility route in this research is Jakarta-Cikampek toll road from KM 47-54. The parameter of this simulation can be seen in Table III.

This simulation consists of two scenarios, node density that varies from 10 to 100 nodes and node velocity that varies from 70 to $120 \mathrm{~km} / \mathrm{h}$. The number of nodes is generated by modifying number nodes configuration of .tcl file inside Network Simulator 2. Each node models a vehicle. For the node velocity scenario, the vehicle speed is modeled using ONE Simulator by setting the minimum and maximum speed of each node. Each node moves based on the mobility route. Each simulation scenario for each configuration consist of five conditions, normal, under black hole attack, under black hole attack with IDS, under jelly fish attack and under jellyfish attack with IDS.

Traffic is modeled as constant bit rate with UDP as transport layer protocol. Total average packet sent is 31011 and total packet received is 29150 for normal condition based on 30 times generated traffic. 
Cite this article as: E. Mustikawati, D. Perdana, and R. M. Negara, "Network Security Analysis in Vanet Against Black Hole and Jelly Fish Attack with Intrusion Detection System Algorithm”, CommIT (Communication \& Information Technology) Journal 11(2), 77-83, 2017.

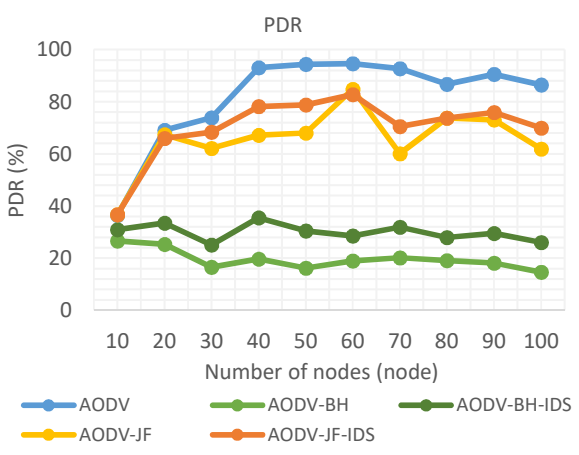

Fig. 3. Packet delivery ratio graph for node density scenario.

\section{Results And Discussions}

\section{A. Node Density Scenario}

In this scenario, the changes to the number of nodes that are used, i.e., $10,20, \ldots, 100$ nodes at $70 \mathrm{~km} / \mathrm{h}$.

These scenarios are simulated in normal condition, under black hole and jellyfish attack, and the addition of IDS algorithm.

Figure 3 shows that the PDR value has some fluctuations but it tends to decrease. Increasing number of nodes causing changes of network topology. Therefore, the routing path is changing for every amount of nodes. AODV shows the graph for normal condition, AODV$\mathrm{BH}$ for black attack condition, AODV-BH-IDS for black hole attack condition with IDS, AODV-JF for jelly fish attack, and AODV-JF-IDS for jelly fish attack with IDS.

Meanwhile, the average value of PDR under black hole attack decreases by $76.13 \%$ from the normal condition. Under jellyfish attack, it decreases by $19.94 \%$ from the normal condition. The black hole attack drops the entire packet in the network, while jellyfish attack still forwards the packet. Graph of the PDR value under jellyfish attack is always greater than black hole attack. It is because IDS algorithm will ignore the first RREP packet and use the second RREP packet arrived to establish the route. For black hole attack with IDS, the PDR value increases by $53.34 \%$ and for jellyfish attack with IDS, it increases by $6.99 \%$.

Figure 4 shows that the throughput value increases along with changes of the number of nodes in all conditions. This is due to increase in the number of nodes. The more packets that are sent and received, the more traffic in the network keeps increasing. Increasing the number of nodes also takes the longer process of route discovery than AODV condition. Each node will look for the best routing by sending an RREQ message to its neighbor nodes and the destination nodes will send RREP message in return and unicast. Therefore, the more the number of nodes in the network is,

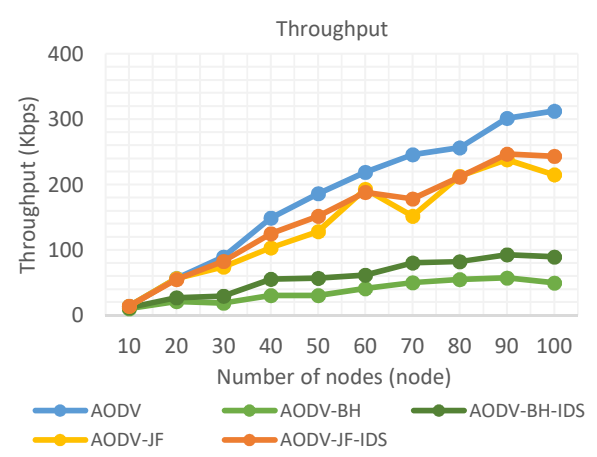

Fig. 4. Throughput graph for node density scenario.

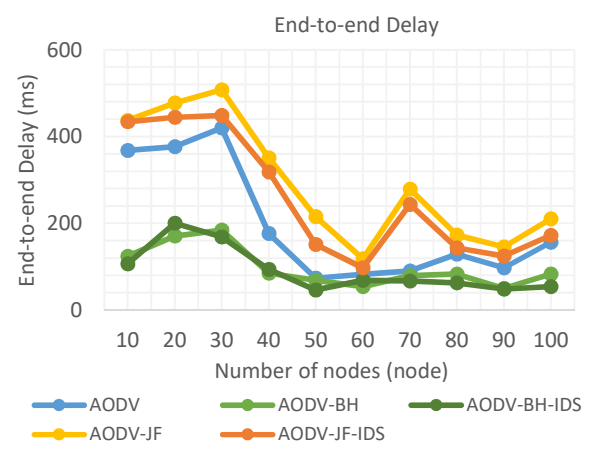

Fig. 5. End-to-end delay graph for node density scenario.

the greater the throughput values on the network is. Black hole and jellyfish attack decrease the throughput value in the network. Under black hole attack, the average of throughput value decreases drastically by $80.18 \%$ or $146.66 \mathrm{Kbps}$ from the normal condition. While under jellyfish attack, the throughput value decreases by $24.28 \%$ or $44.42 \mathrm{Kbps}$ from the normal condition. In other words, black hole attack affects more on throughput parameters compared to jellyfish attack. This is because the black hole node sends a false RREP message to reply to RREQ sender node by aiming to manipulate the routing path. Thus, the malicious node seems to have the latest routing updates by replacing the sequence number into the highest sequence number or 4294967295 and the nearest route by replacing the hop count to 1 . That way, the process of route discovery is faster than normal condition. In other words, exchange routing message is less than the normal condition and the throughput decreases.

Figure 5 shows that the average end-to-end delay value changes in the addition number of nodes. The end-to-end delay value has some fluctuations but it tends to decrease along with addition number of nodes. This fluctuation is due to several factors, namely, traffic data, network topology, and the position of each node. Those things result in fluctuations of the 
Cite this article as: E. Mustikawati, D. Perdana, and R. M. Negara, "Network Security Analysis in Vanet Against Black Hole and Jelly Fish Attack with Intrusion Detection System Algorithm", CommIT

(Communication \& Information Technology) Journal 11(2), 77-83, 2017.

simulations due to routing path that varies each amount of nodes. Under black hole attack, the average delay is decreased by $50.12 \%$ or $98.76 \mathrm{~ms}$ compared to normal conditions. This is because under black hole attack, route discovery process lasts faster than normal condition. The nodes do not need to wait for RREP because the nearest black hole will immediately send a false RREP message. The purpose is to manipulate the other nodes to establish the routing path to a malicious node, and drop all the packets. Therefore, the route discovery process becomes shorter than normal condition. Whereas, the normal condition carries out the process of discovery route in accordance with the mechanism of AODV routing protocol. In other words, the nodes will continue to do the broadcast RREQ to get message with the nearest routing RREP. It indicates that the node's neighbors destination node. Therefore, the average value of end-to-end delay on conditions without any attack is greater than the conditions in the black hole [11, 12].

Jellyfish attack highly affects the value of end-toend delay on the network. The value of end-to-end delay under jelly fish attack is $47.87 \%$. it is greater compared to normal conditions. This is due to jellyfish delay variance attack. The malicious node forwards the data package after delaying few seconds randomly on each packet within 0 to 2 seconds in this research. The value of end-to-end delay under jellyfish attack is higher than other conditions. In other words, jellyfish attack affects more than black hole attack.

The addition of IDS algorithm can reduce end-to-end delay in the offensive black hole and jellyfish attack. Under black hole attack, the average value of end-toend delay is likely to decrease by $16.67 \%$, although a number of nodes value is greater than under black hole attack. Meanwhile, for the jellyfish attack, the average value of end-to-end delay is decreased by $11.50 \%$ compared to under jellyfish attack. The decrease in the value of end-to-end delay is because both attacks sends the false RREP message that aims to take over routing patch to the malicious node itself. So, the data packets will be sent to the malicious node and the attack takes place. By adding the IDS algorithm, the malicious node will perform additional RREP caching mechanism to the routing process to avoid malicious nodes in the network, so the data packets are not forwarding through or stop at the malicious node.

\section{B. Node Velocity Scenario}

In the second scenario, the analysis is carried out based on the change of velocity of the node in several conditions. The change of velocity varies from 70,80 , $90,100,110$, and $120 \mathrm{~km} / \mathrm{h}$. With the same number of

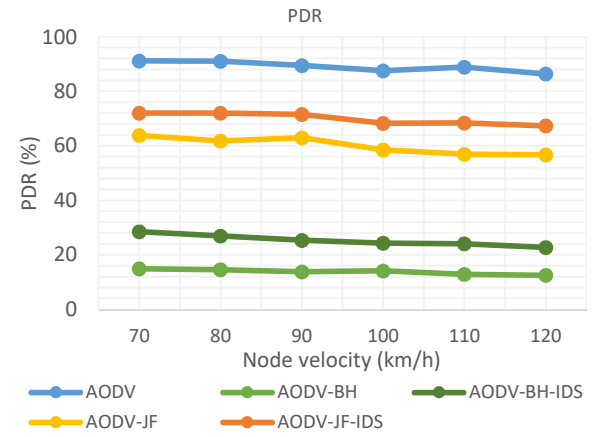

Fig. 6. Packet delivery ratio graph for node velocity scenario.

nodes, i.e., 100 nodes in all conditions and parameters. While, the performance parameters are based on QoS parameters, which is Packet Delivery Ratio (PDR), and throughput, and end-to-end delay.

Figure 6 shows that the value of PDR from all condition has decreased along with the addition of node velocity. Normal conditions have the highest PDR value compared to the other conditions. However, the value of PDR on normal conditions has decreased slightly along with the addition of node velocity. This is due to the faster moving nodes in the network. Then, the possibility of termination of communication links between nodes is getting bigger, so the value of PDR decreases.

In addition, the average of PDR value under black hole attack decreases by $84.53 \%$ from the normal condition. While, in jellyfish attack, the PDR value decreases by $32.5 \%$ from normal conditions. With the node velocity scenario, the termination of node link communication is faster and often occurs so that the packages have not had transmitted the data packets but the link has been disconnected beforehand.

On the conditions of the addition algorithm IDS as a response to the attacks of black hole and jellyfish. For the black hole attack with the addition of IDS algorithm, the average value of PDR increases by $83.69 \%$ from under black hole attack condition. For jellyfish attack with IDS. The PDR value increases by $16.3 \%$ from under attack. The increase in PDR value is due to the RREP caching mechanism in IDS that the first RREP message is ignored and passes through the next RREP arrived message.

Figure 7 shows the throughput value of all conditions tends to decrease along with the addition of node velocity. This happens due to the increasing speed of movement of a node. The termination of the communication link will be more frequent and the process of route discovery becomes disturbed. It can cause a decrease in the throughput value of the 
Cite this article as: E. Mustikawati, D. Perdana, and R. M. Negara, "Network Security Analysis in Vanet Against Black Hole and Jelly Fish Attack with Intrusion Detection System Algorithm”, CommIT (Communication \& Information Technology) Journal 11(2), 77-83, 2017.

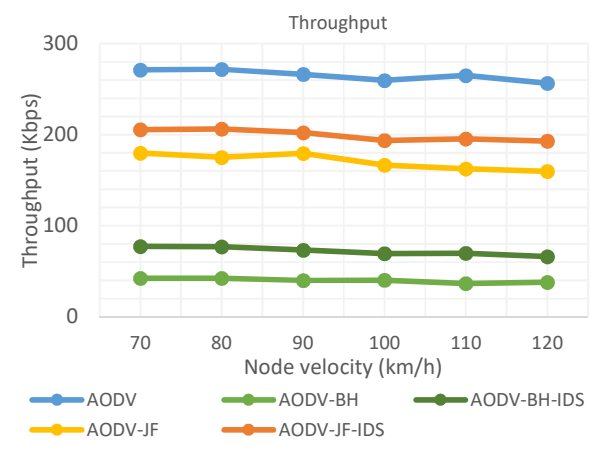

Fig. 7. Throughput graph for node velocity scenario.

network. Black hole attack decreases the throughput value by $84.98 \%$ from normal conditions. Meanwhile, in jellyfish attack, the throughput value decreases by $35.71 \%$. In other words, the black hole attack affects more than jellyfish attack. This is because the black hole attack drops packet, while jellyfish attack still forwards the packet.

On the addition of IDS algorithm, it can be seen that the throughput value increases by $80.79 \%$ of from normal condition. While in jellyfish attack, the throughput value increases by $16.97 \%$ from the normal condition. The addition of IDS algorithm results in increasing the throughput value for both attack, because the algorithm IDS will ignore the first RREP message assuming that it comes from the malicious node and uses the next RREP message arrived as a routing path.

Figure 8 shows that the end-to-end delay graph for all conditions. Overall, the average value of end-to-end delay in all conditions of has decreased along with the increase of velocity. This is because of the increasing speed of nodes, there is the greater possibility of disconnection of communication links between nodes. Under normal conditions, it will result in nodes to look for the latest continuous routing due to network topology change rapidly. Therefore, end-to-end delay value keeps increasing along with the addition of node velocity.

Under black hole attack, the end-to-end delay value has some fluctuations. At speed of $90 \mathrm{~km} / \mathrm{h}$, the end-toend delay value increases, but at speed of $100 \mathrm{~km} / \mathrm{h}$ and $110 \mathrm{~km} / \mathrm{h}$ it decreases. Overall, the end-to-end delay value is decreased by $13.81 \%$ from normal condition, but at the speed of $120 \mathrm{~km} / \mathrm{h}$, it increases by $14.85 \%$ from normal conditions. The black hole node performs the modification process that causes the RREP route discovery to take place faster so that the value of end-to-end delay is much smaller than the normal condition. As the node speed increases the speed, the communication link termination occurs more frequent, so that at a speed of $120 \mathrm{~km} / \mathrm{h}$, the value of end-to-end

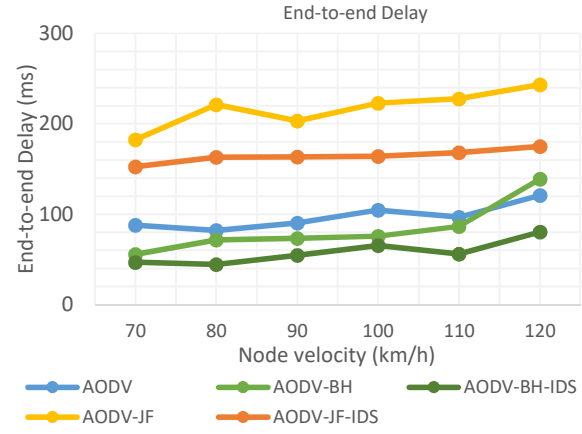

Fig. 8. End-to-end delay graph for the second scenario.

delay is higher than normal conditions.

Under jellyfish attack, the end-to-end delay value increases by $123.19 \%$ from the normal condition and has some fluctuations at speed of $80 \mathrm{~km} / \mathrm{h}$ and $90 \mathrm{~km} / \mathrm{h}$. However, it tends to increase along with the increasing speed of the node. This is because jellyfish nodes wait 0 to $2 \mathrm{~s}$ before forwarding packet to other nodes, so the end-to-end delay value is higher than normal condition.

On the conditions with the addition of algorithm IDS, the value of end-to-end delay has decreased for both attacks. The addition of algorithm IDS on black hole attack can decrease the end-to-end delay value by $30.69 \%$ from black hole condition. Although the value of end-to-end delay on the addition of algorithm still has fluctuations along with the addition of the node speed but it tends to increase along with the increasing speed of node. For the jellyfish attack and IDS algorithm, the end-to-end delay value decreases by $24.18 \%$ from under attack. This happens because in the addition of the IDS, there is an RREP caching mechanism assuming the first RREP packet arrived comes from malicious nodes. Thus, the routing path is established using the next arrived RREP packet.

\section{CONCLUSions}

The addition of IDS algorithm can increase the performance of the network based on the QoS parameters: PDR, throughput, and end-to-end delay compared with conditions under attack. The average values of PDR on the black hole attack increase by $53.34 \%$ in Scenario 1 and $83.69 \%$ in Scenario 2. For jellyfish attack, the average values of PDR increase of $6.99 \%$ in Scenario 1 and $16.3 \%$ in Scenario 2. The average values of throughput on black hole attack increase by $61.24 \%$ or $22.20 \mathrm{kbps}$ on Scenario 1 and $80.79 \%$ or $3.1833 \mathrm{kbps}$ on Scenario 2. For jellyfish attack, average throughput increases by $7.96 \%$ or $11.02 \mathrm{kbps}$ on Scenario 1 and $16.97 \%$ or $28.92 \mathrm{kbps}$ on Scenario 2. The average value of end-to-end delay under black hole attack decreases 
Cite this article as: E. Mustikawati, D. Perdana, and R. M. Negara, "Network Security Analysis in Vanet Against Black Hole and Jelly Fish Attack with Intrusion Detection System Algorithm”, CommIT (Communication \& Information Technology) Journal 11(2), 77-83, 2017.

by $6.53 \%$ or $6.41 \mathrm{~ms}$ on Scenario 1 and $30.69 \%$ or $25.69 \mathrm{~ms}$ in the Scenario 2. For jellyfish attack, the average value of end-to-end delay is decreased by $11.5 \%$ or $33.51 \mathrm{~ms}$ on Scenario 1 and $24.18 \%$ or $52.40 \mathrm{kbps}$ for Scenario 2.

\section{REFERENCES}

[1] A. Muhtadi, D. Perdana, and R. Munadi, "Performance evaluation of aodv, dsdv, and zrp using vehicular traffic load balancing scheme on vanets," International Journal of Simulation System, Science and Technology (IJSSST), pp. 13.113.7, 2015.

[2] B. Heru, B. Benny, D. Defendy, and W. Hento, "Keamanan jaringan menggunakan unified threat management pada server berbasiskan linux," CommIT (Communication and Information Technology) Journal, vol. 1, no. 1, pp. 48-59, 2007.

[3] D. Perdana, M. Nanda, R. Ode, and R. F. Sari, "Performance evaluation of puma routing protocol for manhattan mobility model on vehicular ad-hoc network," in Telecommunications (ICT), 2015 22nd International Conference. IEEE, 2015, pp. 80-84.

[4] R. S. Raw, M. Kumar, and N. Singh, "Security challenges, issues and their solutions for vanet," International Journal of Network Security \& Its Applications (IJNSA), vol. 5, no. 5, 2013.

[5] K. S. Nisha and S. K. Arora, "Analysis of black hole effect and prevention through ids in manet," American Journal of Engineering Research (AJER), vol. 2, no. 10, pp. 214-220, 2013.

[6] H. R. Khirasariya, "Simulation study of jellyfish attack in manet using aodv routing protocol,"
Journal of Information, Knowledge, and Research in Computer Engineering, vol. 2, no. 2, pp. 344347, 2013.

[7] L. Wenshuang, L. Zhuorong, Z. Hongyang, W. Shenling, and B. Rongfang, "Vehicular ad hoc networks: Architectures, research issues, methodologies, challenges, and trends," International Journal of Distributed Sensor Networks, vol. 11, no. 8, pp. 1-11, Jan. 2015.

[8] L. Mamta and P. Sharda Prasad, "Simulation of blackhole attack," International Journal of Engineering Technology \& Management Research (IJETMR), vol. 2, no. 2, pp. 23-30, 2014.

[9] A. K. Gupta, N. Kaur, and A. Kaur, "A survey on behaviour of aodv and olsr routing protocol of manets under black hole attack," International Journal of Computer Science \& Technology, vol. 2, no. 4, pp. 349-352, 2011.

[10] S. Dokurer, "Simulation of black hole attack in wireless ad-hoc network," Master's thesis, Ankara, Atilim University, 2006.

[11] V. Bibhu, R. Kumar, B. S. Kumar, and D. K. Singh, "Performance analysis of black hole attack in vanet," International Journal of Computer Network and Information Security, vol. 4, no. 11, pp. 47-54, 2012.

[12] B. Cherkaoui, A. Beni-hssane, and M. Erritali, "A clustering algorithm for detecting and handling black hole attack in vehicular ad hoc networks," in Advances in Intelligent Systems and Computing. Springer, 2017, pp. 481-490. 